\title{
miR-1301/TRIAP1 Axis Participates in Epirubicin-Mediated Anti-Proliferation and Pro-Apoptosis in Osteosarcoma
}

\author{
Lijun $\mathrm{Yu}^{1 *}$, Min Meng ${ }^{1 *}$, Yun Bao ${ }^{1}$, Chao Zhang ${ }^{2}$, Bei Gao ${ }^{1}$, Rina Sa ${ }^{1}$, and Wenyuan $\mathrm{Luo}^{2}$ \\ ${ }^{1}$ Department of Pharmacy and ${ }^{2}$ Department III of Orthopedic, Gansu Provincial Hospital, Lanzhou, Gansu, China.
}

\begin{abstract}
Purpose: Epirubicin is one of the most effective drugs against osteosarcoma. miR-1301 is involved in the occurrence and development of osteosarcoma. Whether miR-1301 is responsible for the chemosensitivity of osteosarcoma cells to epirubicin remains largely unknown.

Materials and Methods: U2OS and SAOS-2 cells were treated with various concentrations of epirubicin. Flow cytometry was employed to evaluate cell apoptotic rate. Cell proliferation was measured by Cell Counting Kit-8 assay. Western blot and quantitative real-time polymerase chain reaction were utilized to detect the expressions of B-cell lymphoma-2 (Bcl-2), Bcl-2 assaciated X protein (Bax), cleaved-caspase-3, cleaved-poly (ADP-ribose) polymerases (PARP1), TP53-regulated inhibitor of apoptosis 1 (TRIAP1), and microRNA-1301 (miR-1301). The relationship between miR-1301 and TRIAP1 was determined by luciferase reporter assay.

Results: Epirubicin inhibited proliferation in a dose-dependent manner, induced apoptosis, decreased the expression of Bcl-2, and increased the expressions of Bax, cleaved-caspase-3, and cleaved-PARP1 in osteosarcoma cells. miR-1301 was downregulated in U2OS and SAOS-2 cells. Importantly, epirubicin significantly increased the levels of miR-1301. Overexpression of miR-1301 suppressed proliferation and promoted apoptosis. Interestingly, those effects were enhanced by epirubicin. In contrast, miR-1301 depletion attenuated the epirubicin-mediated anti-osteosarcoma effect. miR-1301 negatively regulated the expression of TRIAP1 in U2OS and SAOS-2 cells. Furthermore, epirubicin inhibited the mRNA and protein levels of TRIAP1 by upregulating miR-1301 levels. Epirubicin suppressed cell proliferation by downregulating TRIAP1.

Conclusion: miR-1301 was implicated in the chemosensitivity of osteosarcoma to epirubicin by modulating TRIAP1.
\end{abstract}

Key Words: miR-1301, TRIAP1, epirubicin, osteosarcoma

\section{INTRODUCTION}

Osteosarcoma is a malignant and rare tumor that primarily affects bones in juveniles and adults. ${ }^{1,2}$ It has been reported that osteosarcoma patients with metastatic or recurrent dis-

Received: February 19, 2019 Revised: May 25, 2019

Accepted: June 13, 2019

Corresponding author: Wenyuan Luo, MD, Department III of Orthopedic, Gansu Provincial Hospital, No. 204, West Donggang Road, Chengguan District, Lanzhou 730000, Gansu, China.

Tel: 86-0931-8281007, Fax: 86-0931-8281007, E-mail: budalagong305@sina.com

*Lijun Yu and Min Meng contributed equally to this work.

-The authors have no potential conflicts of interest to disclose.

(C) Copyright: Yonsei University College of Medicine 2019

This is an Open Access article distributed under the terms of the Creative Commons Attribution Non-Commercial License (https://creativecommons.org/licenses/ by-nc/4.0) which permits unrestricted non-commercial use, distribution, and reproduction in any medium, provided the original work is properly cited. ease fare poorly, with overall survival rates of less than $20 \% .^{3}$ Therapeutic strategies, especially chemotherapy, lead to improved survival and outcomes in patients with osteosarcoma. ${ }^{4-6}$ Epirubicin is one of the most effective drugs against cancer and has gained widespread use in multiple cancers. ${ }^{7,8}$ Moreover, studies have shown that epirubicin could enhance the antitumor activity in osteosarcoma., ${ }^{9,10}$ Therefore, combination chemotherapy with epirubicin and other agents may provide an effective approach for osteosarcoma patients.

MicroRNAs (miRNAs), a class of small noncoding RNAs, can act as tumor-suppressors in tumorigenesis and development. ${ }^{11}$ Research has revealed that inhibition of miR-598 expression stimulates proliferation, migration, and invasion in osteosarcoma. Also, restoration of miR125a5p results in repressing osteosarcoma cell migration, invasion and epithelialmesenchymal transition by targeting matrix metallopeptidase-11. ${ }^{12}$ 
Moreover, it is well documented that miRNAs are involved in chemotherapy of cancers. ${ }^{13,14} \mathrm{Li}$, et al. ${ }^{15}$ noted that a combination of miR-19a and miR-205 in the serum may predict chemosensitivity to epirubicin plus paclitaxel neoadjuvant chemotherapy for the luminal a subtype of breast cancer. Furthermore, miR-671-5p has been found to sensitize breast cancer cells to cisplatin, 5-fluorouracil, and epirubicin exposure and to reduce DNA repair capability in post-drug exposed breast cancer cells. ${ }^{16}$ Zhang, et al. ${ }^{17}$ found that miR-4443 depletion increases the sensitivity of epirubicin-resistant H1299 cells to epirubicin by decreasing the IC50 of epirubicin and inducing cell apoptosis and G0/G1 cell cycle arrest in non-small cell lung cancer. Additionally, previous studies have demonstrated that miRNAs are also correlated with chemosensitivity in osteosarcoma. ${ }^{18}$ For example, miR-125b was found to enhance chemosensitivity to cisplatin in osteosarcoma. ${ }^{19}$ miR-302b increased epirubicin-mediated anti-proliferation and pro-apoptosis effect in osteosarcoma. ${ }^{20}$ Recently, miR-1301 was reported to inhibit migration and invasion by targeting B-cell chronic lymphocytic leukemia/lymphoma 9 in osteosarcoma. ${ }^{21}$ However, whether miR-1301 is responsible for anti-osteosarcoma effects remains to be elucidated. TP53-regulated inhibitor of apoptosis 1 (TRIAP1) is overexpressed in many cancers and acts as an oncogene in tumorigenesis and development. ${ }^{22}$ Nevertheless, still relatively little is known about its role in osteosarcoma.

In this study, we hypothesized that TRIAP1 may be a target of miR-1301 and investigated the role of miR-1301/TRIAP1 in epirubicin-mediated anti-osteosarcoma effects.

\section{MATERIALS AND METHODS}

\section{Cell culture and treatment}

Human osteoblast hFOB1.19 cells and human osteosarcoma U2OS and SAOS-2 cells were purchased from American Type Culture Collection (Manassas, VA, USA) and cultured in the Dulbecco's Modified Eagle's Medium (Thermo Fisher Scientific, Waltham, MA, USA) supplemented with $10 \%$ fetal bovine serum (Thermo Fisher Scientific) and 1\% penicillin/streptomycin stock solution (Beyotime, Shanghai, China). Cells were maintained in a humidified atmosphere containing $5 \% \mathrm{CO}_{2}$ at $37^{\circ} \mathrm{C}$. U2OS and SAOS- 2 cells ( $80 \%$ confluence in 6 -well plates) were treated with different concentrations $(0,0.1,0.25,0.5,1$, $2.5,5$, or $10 \mu \mathrm{g} / \mathrm{mL}$ ) of epirubicin (MedChemExpress, Princeton, NJ, USA) for 24 or $48 \mathrm{~h}$. U2OS and SAOS-2 cells were transfected with miR-NC mimics, miR-1301 mimics, miR-NC inhibitors, miR-1301 inhibitors, pcDNA vector (vector), and TRIAP1 overexpression plasmid (TRIAP1). After 24-h transfection, cells were treated with epirubicin $(1 \mu \mathrm{g} / \mathrm{mL})$ for $48 \mathrm{~h}$. The transfected or treated cells were used for subsequent experiments.

\section{qRT-PCR assay}

TRIzol (Beyotime) was introduced to extract total RNAs from cells according the manufacturer's instructions. Then, RNA was reversely transcribed into complementary DNA using TaqMan MicroRNA Reverse Transcription Kits (Biosystems, Foster City, CA, USA) or TIANScript RT Kits (Baolebo biotech, Beijing, China). Quantitative real-time polymerase chain reaction (qRT-PCR) analyses were carried out using Power SYBR Green kits (Takara, Shiga, Japan). The primers were as follows: TRIAP1 forward, 5'-AGGATTTCGCAAGTCCAGAA-3', and reverse, 5'-GCTGATTCCACCCAAGTAT-3'; glyceraldehyde3-phosphate dehydrogenase (GAPDH) forward, 5'-AGAAGG CTGGGGCTCATTTG-3', and reverse, 5'-AGGGGCCATCCA CAGTCTTC-3'; miR-1301 forward, 5'-ACACTCCAGCTGGG TTGCAGCTGCCTGGGAGTGA-3', and reverse, 5'-CTCAACT GGTGTCGTGGA- 3'; U6 forward, 5'-CTTCGGCAGCACAT ATAC-3' and reverse, 5'-GAACGCTTCACGAATTTGC-3'. The relative expression levels of miRNA (normalized to U6 small nuclear RNA) and mRNA (normalized to GAPDH) were evaluated by the $2^{-\Delta \Delta \mathrm{Ct}}$ method.

\section{CCK-8 assay}

Osteosarcoma cells (5000 cells/well) were seeded in 96-well plates and detected for cell proliferation using Cell Counting Kit-8 (CCK-8) assay according to the manufacturer's instructions. Briefly, the cells were incubated with $10 \mu \mathrm{L}$ of CCK-8 (AbMole BioScience, Houston, TX, USA) at $37^{\circ} \mathrm{C}$ for $4 \mathrm{~h}$. Absorbance at $450 \mathrm{~nm}$ was evaluated using a microtiter plate reader (Thermo Fisher Scientific). Each group was tested with three replicates.

\section{Cell apoptosis assay}

Cell apoptotic rate was evaluated as described in a previous study using Annexin V-PE/7-AAD apoptosis assay (KeyGEN BioTECH, Nanjing, China).$^{20}$ Briefly, cells were washed with cooled phosphate buffer saline and trypsinized. Annexin V-PE $(1 \mu \mathrm{L})$ and 7 -AAD $(5 \mu \mathrm{L})$ were added in a dark room for $10 \mathrm{~min}$ incubation. Cell apoptotic rate was detected by a FACSCalibur flow cytometer with Cell Quest software (BD Biosciences, Franklin Lakes, NJ, USA).

\section{Western blot assay}

Cells were collected in Radio Immunoprecipitation Assay lysis buffer containing protease inhibitors (Beyotime). Cell lysates were centrifuged, and supernatants were collected and mixed with $6 \times$ loading buffer and boiled for $10 \mathrm{~min}$. Proteins were quantified using bicinchoninic acid Protein Assay Kits (Solarbio, Beijing, China), separated with sodium dodecyl sulfate polyacrylamide gel, and transfected onto polyvinylidene fluoride membranes (Millipore, Billerica, MA, USA). The primary antibodies used in the present study were anti-Bcl-2: ab32124, anti-Bax: ab32503, anti-cleaved-caspase-3: ab2302, anti-cleaved-poly (ADP-ribose) polymerase-1 (anti-cleaved- 
PARP1): ab32064, anti-TRIAP1: ab225938, and anti-GAPDH: ab181602, which were purchased from Abcam (Cambridge, MA, USA). Horseradish peroxidase conjugated-secondary anti-body (goat anti-rabbit IgG: ab150077) was obtained from Cell Signaling Technology (Massachusetts, MA, USA). The protein signals were visualized using enhanced chemiluminescence (Thermo Fisher Scientific). Densitometry values were normalized to levels of GAPDH and analyzed using Image $1.48 \mathrm{v}$ software (National Institutes of Health, Bethesda, MD, USA).

\section{Luciferase reporter assay}

The 3'-UTR of TRIAP1 containing predicted miR-1301 binding sites [wild-type TRIAP1 (WT) and mutant TRIAP1 (MUT)] were subcloned into the pmirGLO vector (Promega, Madison, WI, USA). Then, U2OS and SAOS-2 cells were cultured in 24-well plates and co-transfected with TRIAP1-WT or TRIAP1-MUT and miR-1301 mimics or miR-NC mimics, respectively. The relative luciferase activities were measured using the Dual-Luciferase Reporter Assay System (Promega).

\section{Statistical analysis}

All data are expressed as a mean \pm standard deviation. Statisti- cal analysis was conducted using SPSS 18.0 software (SPSS, Inc., Chicago, IL, USA). Results were considered statistically significant only if the $p$ value was less than 0.05 using Student's t test or one-way ANOVA. Our research was approved by Gansu Provincial Hospital.

\section{RESULTS}

\section{Effects of epirubicin on proliferation and apoptosis in osteosarcoma cells}

First, to determine the effect of epirubicin on osteosarcoma cells, U2OS and SAOS-2 cells were treated with epirubicin at various concentrations. The results of CCK-8 assays showed that epirubicin inhibited osteosarcoma cell proliferation in a dose-dependent manner (Fig. 1A). Furthermore, $1 \mu \mathrm{g} / \mathrm{mL}$ of epirubicin was regarded as a standard concentration, since it provided effective inhibition of cell viability and a good amount of surviving cells. Then, the effect of epirubicin on cell apoptosis was further evaluated. We demonstrated that cell apoptotic rate was higher in cells with epirubicin exposure than that of cells treated with $0 \mu \mathrm{g} / \mathrm{mL}$ of epirubicin (Fig. 1B). More-
A

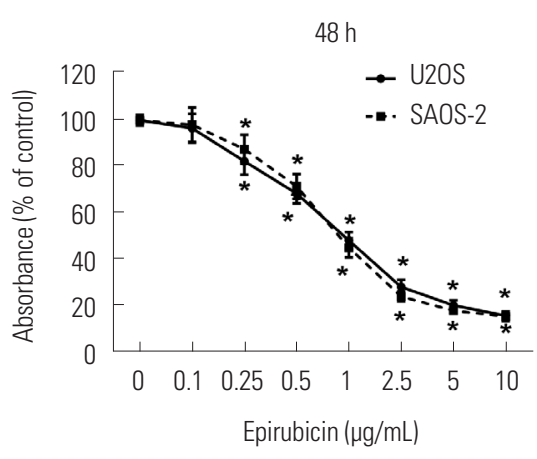

C

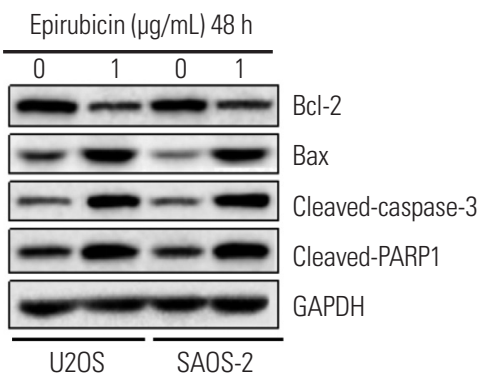

B

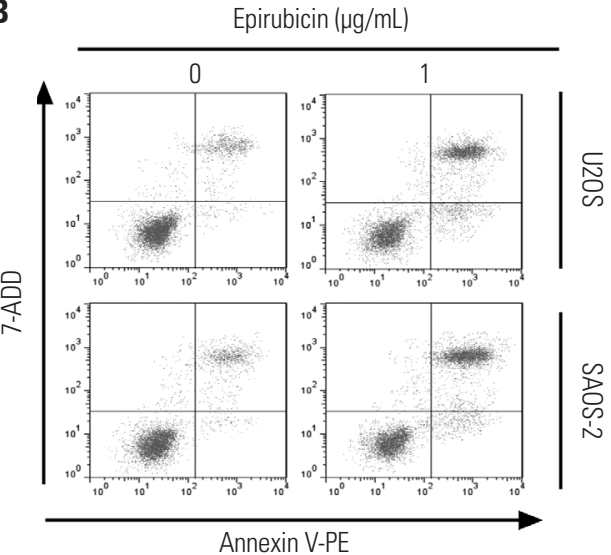

U20S

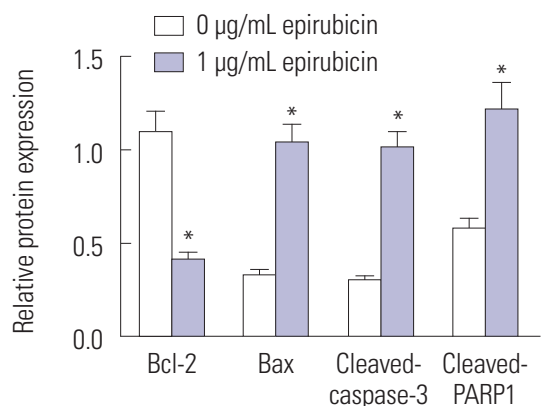

$48 \mathrm{~h}$

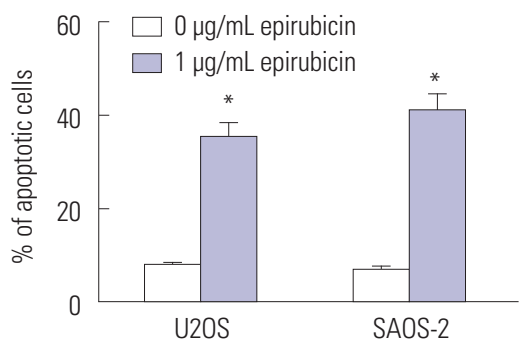

SAOS-2

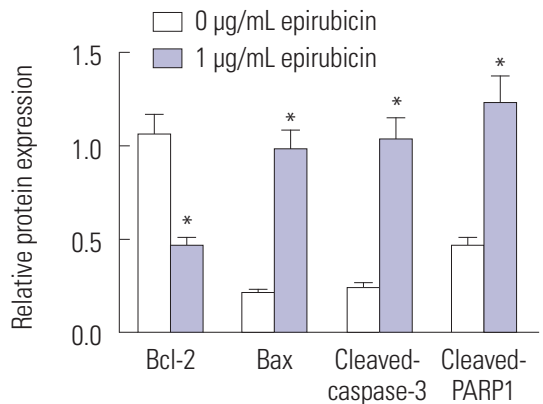

Fig. 1. Effects of epirubicin on proliferation and apoptosis in osteosarcoma cells. (A) Osteosarcoma cell lines U2OS and SAOS-2 were treated with different concentrations of epirubicin for $48 \mathrm{~h}$, and Cell Counting Kit-8 assay was performed to evaluate cell proliferation. (B) U20S and SA0S-2 cells were exposed to $0 \mu \mathrm{g} / \mathrm{mL}$ or $1 \mu \mathrm{g} / \mathrm{mL}$ of epirubicin for $48 \mathrm{~h}$, respectively. Flow cytometry was introduced to determine cell apoptotic rate. (C) Western blot was conducted to examine the expressions of Bcl-2, Bax, cleaved-caspase-3, and cleaved-poly (ADP-ribose) polymerase-1 (cleaved-PARP1) in U20S and SAOS-2 cells with $48 \mathrm{~h}$ of $0 \mu \mathrm{g} / \mathrm{mL}$ or $1 \mu \mathrm{g} / \mathrm{mL}$ epirubicin exposure. ${ }^{*} p<0.05$. Bcl-2, B-cell lymphoma-2; Bax, Bcl-2 assaciated X protein; GAPDH, glyceraldehyde-3-phosphate dehydrogenase. 
over, the detailed molecular mechanisms of epirubicin affecting apoptosis were subsequently explored. The level of antiapoptosis protein Bcl-2 was decreased, while the expressions of Bax, cleaved-caspase- 3 and cleaved-PARP1 were increased in U2OS and SAOS-2 cells treated with $1 \mu \mathrm{g} / \mathrm{mL}$ of epirubicin, compared with cells without epirubicin treatment (Fig. 1C), indicating that epirubicin participates in pro-apoptosis and anti-proliferation in osteosarcoma in vitro.

\section{Epirubicin stimulates the expression of miR-1301 in U2OS and SAOS-2 cells}

Then, we continued to examine the levels of miR-1301 in osteosarcoma cells. We observed that miR-1301 was downregulated in U2OS and SAOS-2 cells, compared with that of hFOB1.19 human osteoblast cells (Fig. 2A), suggesting that miR-1301 may act as a tumor suppressor in osteosarcoma. Moreover, whether epirubicin regulates the expression of miR-1301 was further addressed. As expected, epirubicin increased the abundance of miR-1301 in U2OS and SAOS-2 cells relative to that of the Ctrl group (Fig. 2B), demonstrating that miR-1301 may play a vital role in epirubicin-mediated effects in osteosarcoma cells.

\section{Upregulation of miR-1301 contributes to the} anti-cancer effect of epirubicin in osteosarcoma

Considering that the level of miR-1301 was increased in osteosarcoma cells exposed to epirubicin, the role of miR-1301 in epirubicin-mediated anti-osteosarcoma was subsequently investigated. The results of transfection efficiency revealed that the introduction of miR-1301 mimics and epirubicin alone induced the enforced expression of miR-1301. Moreover, the promotion effect was enhanced by combination of epirubicin and miR-1301 mimics (Fig. 3A). As shown in Fig. 3B, the data further demonstrated that cell proliferation was impeded in the miR-1301 mimics group. More importantly, the inhibition effect was strengthened by a combination of them. Furthermore, the results of flow cytometry presented that cell apoptotic rate was elevated in cells transfected with miR-1301 mimics or treated with epirubicin. Intriguingly, a combination of miR1301 overexpression and epirubicin reinforced the boosted effect on cell apoptosis (Fig. 3C). In addition, the expressions of apoptosis-related proteins were also analyzed in U2OS and SAOS-2 cells using Western blot. Our data showed that the combination of epirubicin and miR-1301 mimics increased the effects observed with separate treatment of them on the expressions of apoptosis-related protein (Fig. 3D), which was consistent with the data of flow cytometry. Consequently, our findings demonstrated that miR-1301 contributes to the epirubicin-mediated anti-osteosarcoma effect via a regulatory effect on cell proliferation and apoptosis.

\section{MiR-1301 depletion weakens the anti-cancer effect of epirubicin in osteosarcoma}

Based on the above results, we further conducted cell transfection to examine whether miR-1301 depletion weakens the anti-osteosarcoma effect of epirubicin. As displayed in Fig. $4 \mathrm{~A}$, the transfection of miR-1301 inhibitors abolished epirubicin-induced miR-1301 promotion. Simultaneously, cell proliferation and apoptosis continued to be evaluated. Downregulation of miR-1301 abated the epirubicin-mediated inhibition of cell proliferation in U2OS and SAOS-2 cells (Fig. 4B). Moreover, epirubicin promoted cell apoptosis, which was undermined by downregulation of miR-1301 (Fig. 4C). To expound on this observation, Western blot analysis of apoptosis-related proteins was also performed (Fig. 4D). The data further verified that miR-1301 knockdown overturned the pro-apoptosis effect of epirubicin in osteosarcoma cells.

\section{TRIAP1 is a target of miR-1301}

In order to explore the molecular mechanisms of miR-1301 involvement in epirubicin-mediated anti-osteosarcoma effects, we used the TargetScan online database to search for potential candidate targets of miR-1301 and found that TRIAP1 contained binding sites with miR-1301 (Fig. 5A). More-
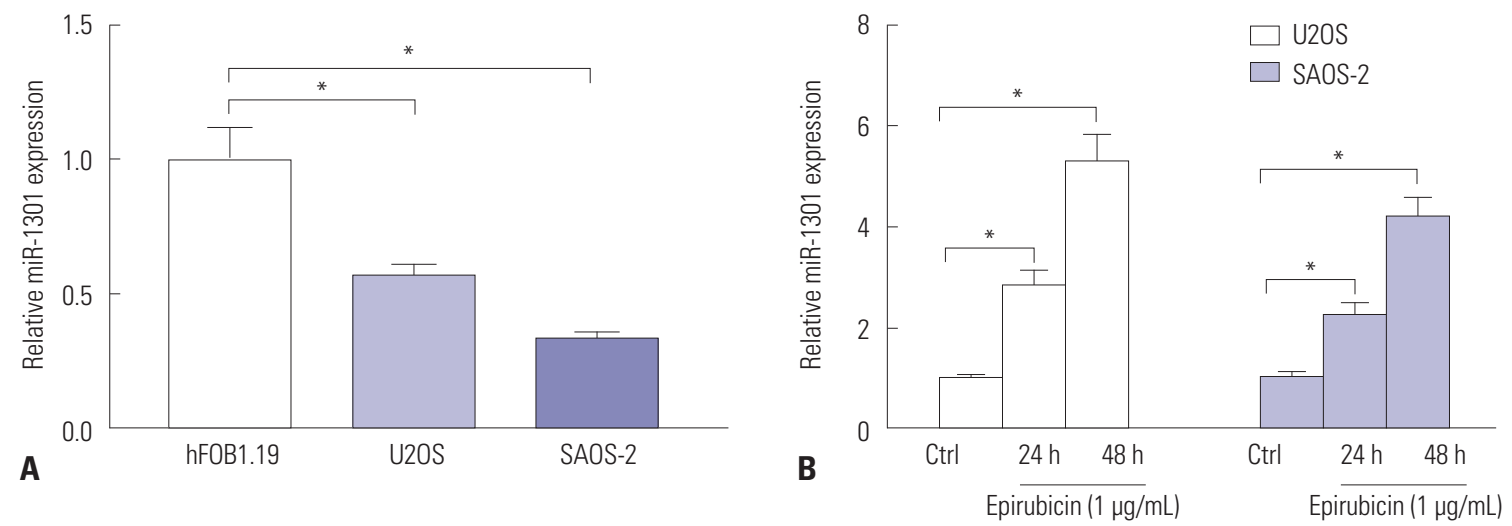

Fig. 2. Epirubicin stimulates the expression of microRNA-1301 (miR-1301) in U20S and SAOS-2 cells. (A) qRT-PCR was employed to measure the expression of miR-1301 in human osteoblast cells hFOB1.19, U20S cells, and SAOS-2 cells. (B) The expression of miR-1301 in U20S or SAOS-2 cells treated with $1 \mu \mathrm{g} / \mathrm{mL}$ of epirubicin for $24 \mathrm{~h}$ or $48 \mathrm{~h} .{ }^{*} p<0.05$. qRT-PCR, quantitative real-time polymerase chain reaction. 
A

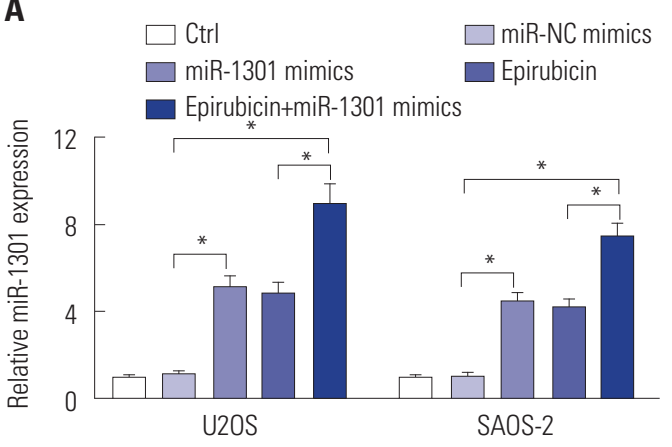

B
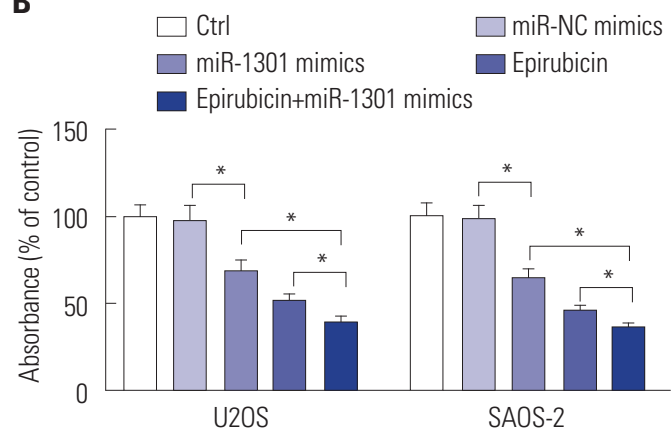

Epirubicin+

C Ctrl miR-NC mimics miR-1301 mimics Epirubicin miR-1301 mimics
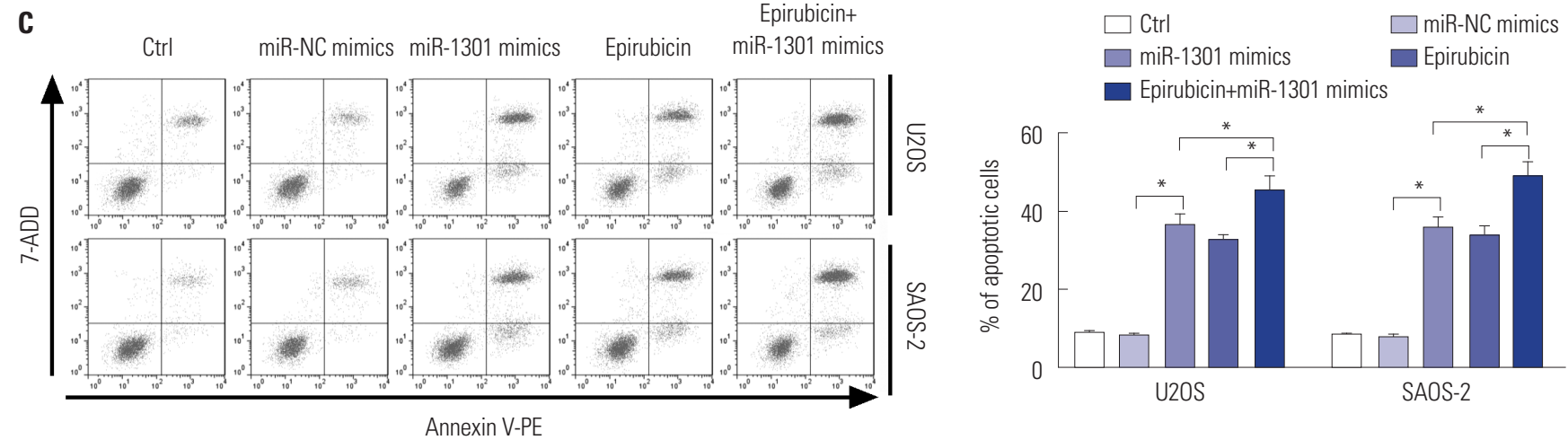

D

Epirubicin miR-NC mimics miR-1301 mimics

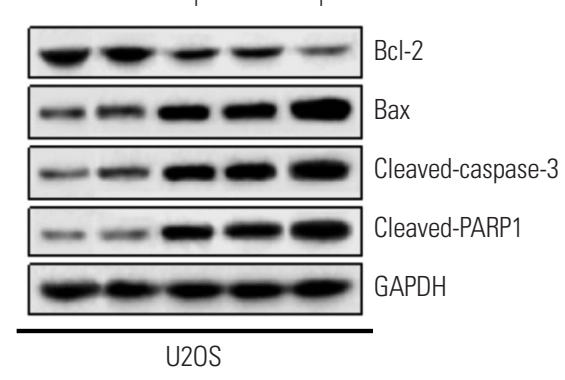

Epirubicin miR-NC mimics miR-1301 mimics

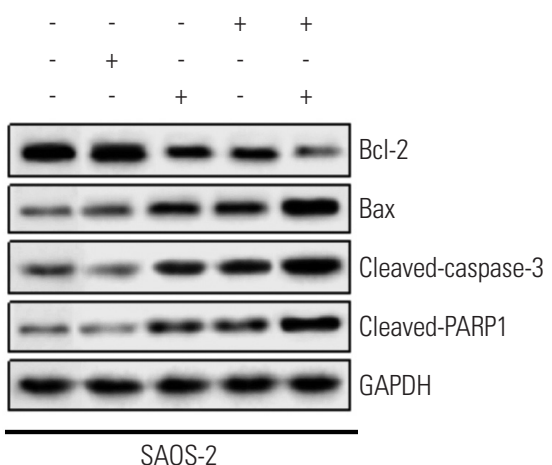

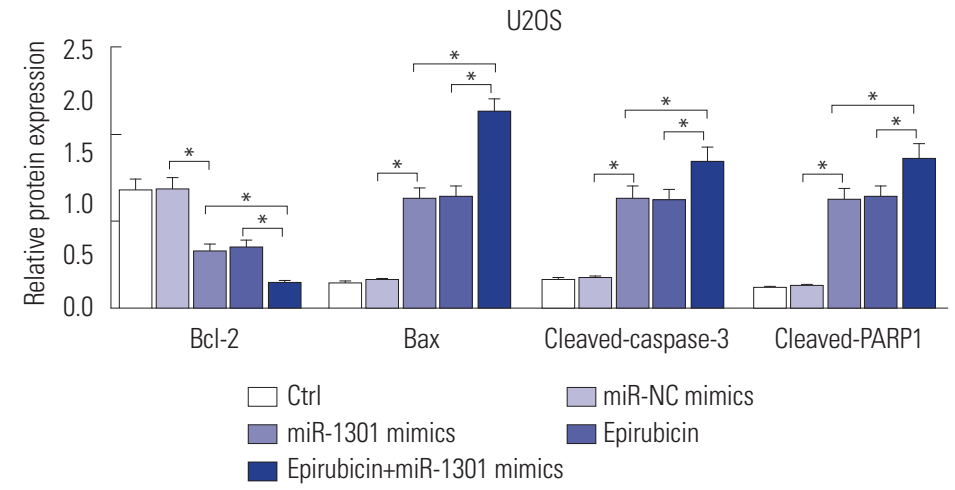

SAOS-2

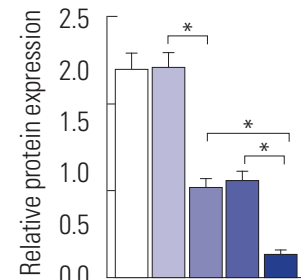

Bcl-2

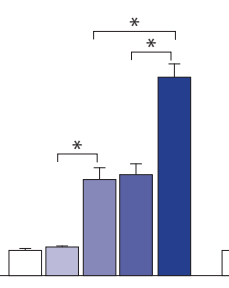

Bax

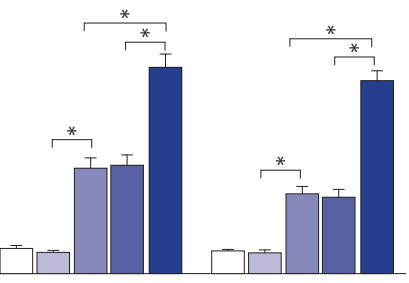

Cleaved-caspase-3 Cleaved-PARP1

$$
\begin{aligned}
& \square \text { Ctrl } \quad \square \text { miR-NC mimics } \\
& \square \text { miR-1301 mimics } \square \text { Epirubicin } \\
& \square \text { Epirubicin+miR-1301 mimics }
\end{aligned}
$$

Fig. 3. Upregulation of microRNA-1301 (miR-1301) contributes to the anti-cancer effect of epirubicin in osteosarcoma. U2OS and SAOS-2 cells were transfected with miR-NC mimics or miR-1301 mimics, and after 24-h transfection, cells were treated with epirubicin $(1 \mu \mathrm{g} / \mathrm{mL})$ for $48 \mathrm{~h}$. Cells were divided into five groups: Ctrl, miR-NC mimics, miR-1301 mimics, Epirubicin, or Epirubicin+miR-1301 mimics. (A) qRT-PCR was introduced to detect the levels of miR1301. (B) Cell Counting Kit-8 was applied to analyze cell proliferation in U2OS and SAOS-2 cells. (C) Flow cytometry was utilized to evaluate cell apoptotic rate. (D) The expressions of Bcl-2, Bax, cleaved-caspase-3, and cleaved-poly (ADP-ribose) polymerase-1 (cleaved-PARP1) in U20S and SA0S-2 cells were measured using Western blot. The + respensents Epirubicin or mimic was used to treat cells, while the meaning of - was opposite with $+{ }^{*} p<0.05$. Bcl-2, B-cell lymphoma-2; Bax, Bcl-2 assaciated X protein; GAPDH, glyceraldehyde-3-phosphate dehydrogenase. 
A

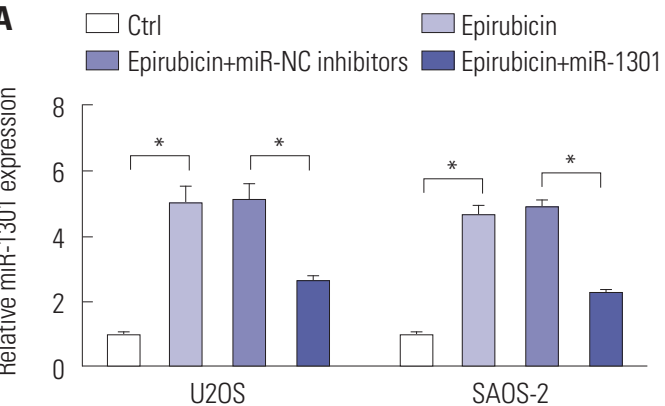

B

$\square$ Ctrl $\square$ Epirubicin

$\square$ Epirubicin+miR-NC inhibitors $\square$ Epirubicin+miR-1301 inhibitors

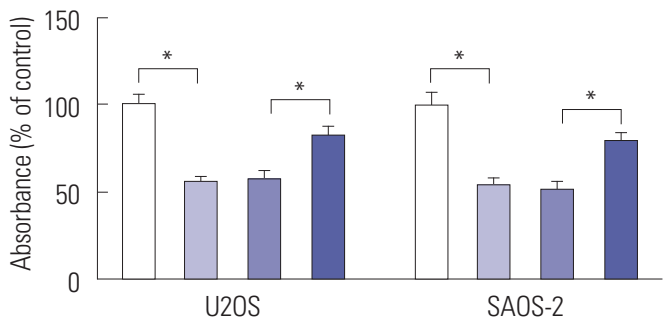

C

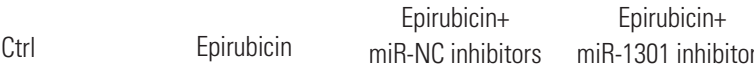
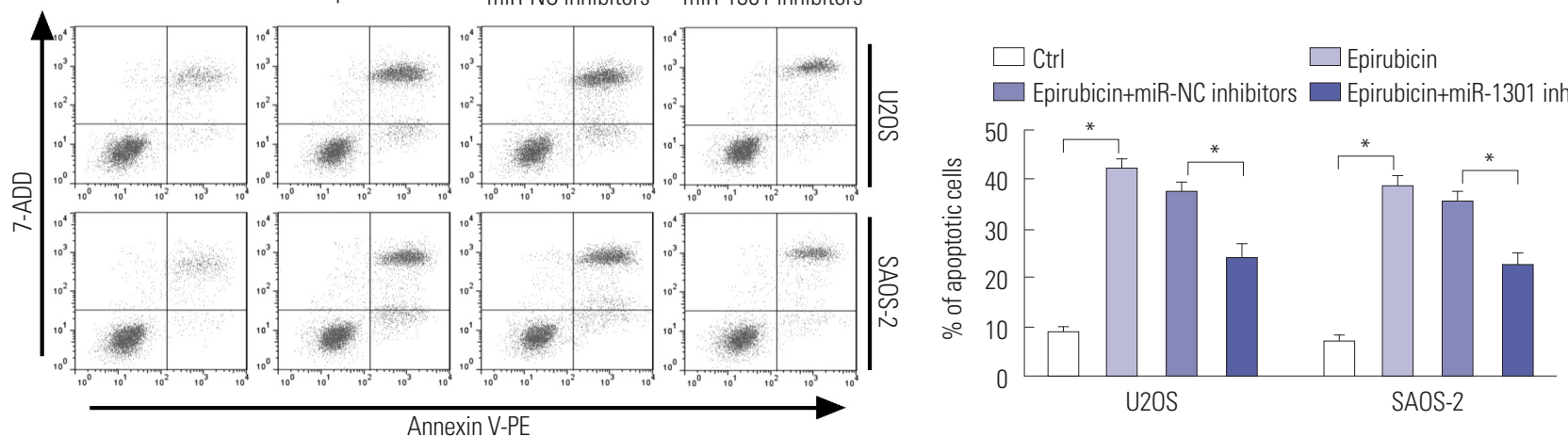

D

Epirubicin miR-NC inhibitors miR-1301 inhibitors

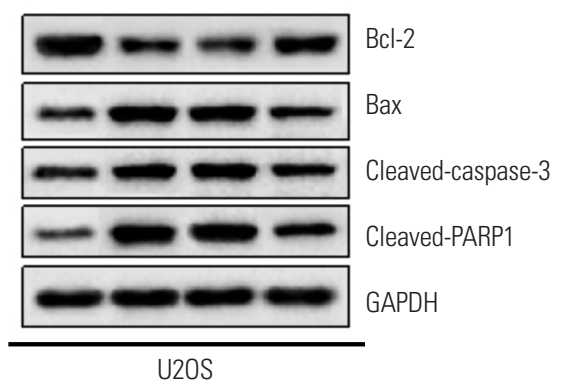

Epirubicin miR-NC inhibitors miR-1301 inhibitors

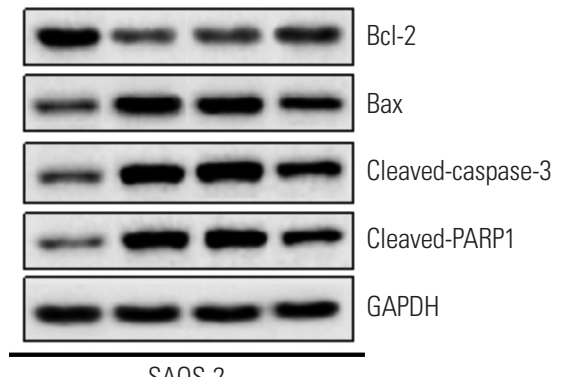

SAOS-2
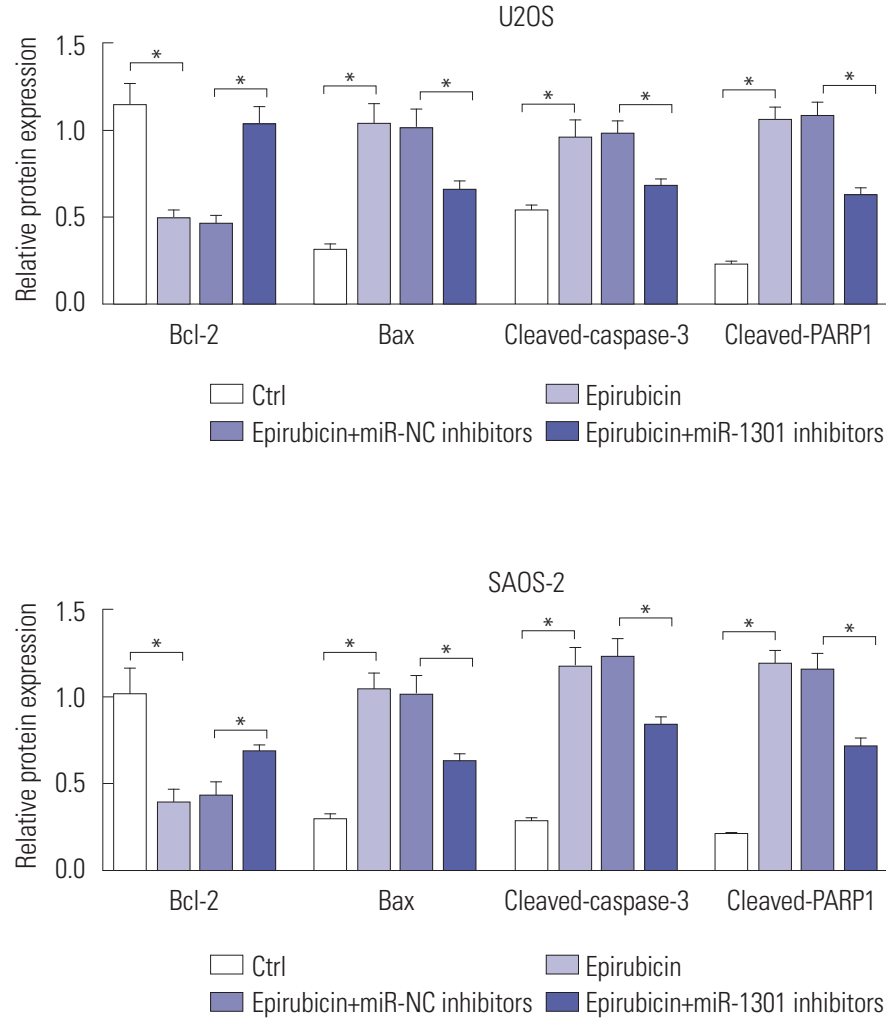

Fig. 4. MicroRNA-1301 (MiR-1301) depletion weakens the anti-cancer effect of epirubicin in osteosarcoma. U2OS and SAOS-2 cells were transfected with miR-NC inhibitors or miR-1301 inhibitors, and after 24-h transfection, cells were treated with epirubicin $(1 \mu \mathrm{g} / \mathrm{mL})$ for $48 \mathrm{~h}$. Cells were divided into four groups: Ctrl, Epirubicin, Epirubicin+miR-NC inhibitors, or Epirubicin+miR-1301 inhibitors. (A) The expression levels of miR-1301 in cells were determined using qRT-PCR. (B) Cell proliferation was measured in U2OS and SAOS-2 cells. (C) Flow cytometry was utilized to analyze the cell apoptotic rate. (D) Western blot was employed to examine the expressions of Bcl-2, Bax, cleaved-caspase-3, and polymerases (PARP1) in U2OS and SAOS-2 cells. The + respensents Epirubicin or mimic was used to treat cells, while the meaning of - was opposite with $+.{ }^{*} p<0.05$. Bcl-2, B-cell lymphoma-2; Bax, Bcl-2 assaciated X protein; GAPDH, glyceraldehyde-3-phosphate dehydrogenase. 
A Position 604-611 of TRIAP1 3' UTR

$\begin{array}{lrrr}\text { TRIAP1-WT } & 5^{\prime} & \text {..AGAUUAAGAUGUGAAAGCUGCAA... } & 3^{\prime} \\ \text { miR-1301 } & 3^{\prime} & \text { CUUCAGUGAGGGUCCGUCGACGUU } & 5^{\prime} \\ \text { TRIAP1-MUT } & 5^{\prime} & \text {...AGAUUAAGAUGUGAACCUGAAGC... } & 3^{\prime}\end{array}$

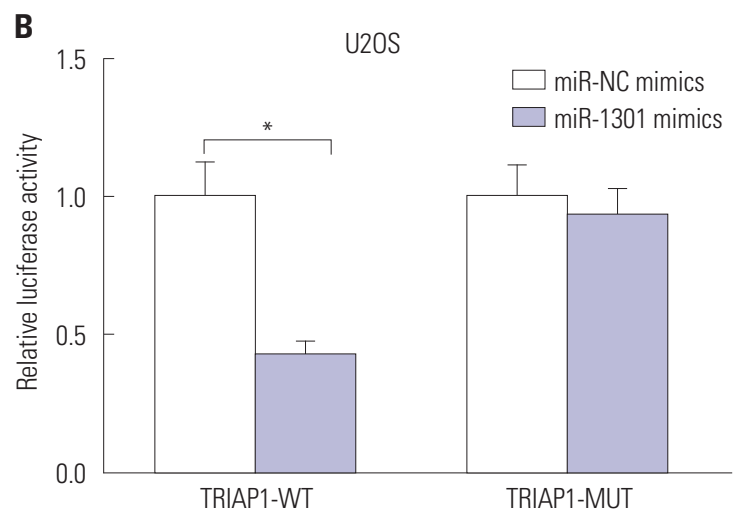

C
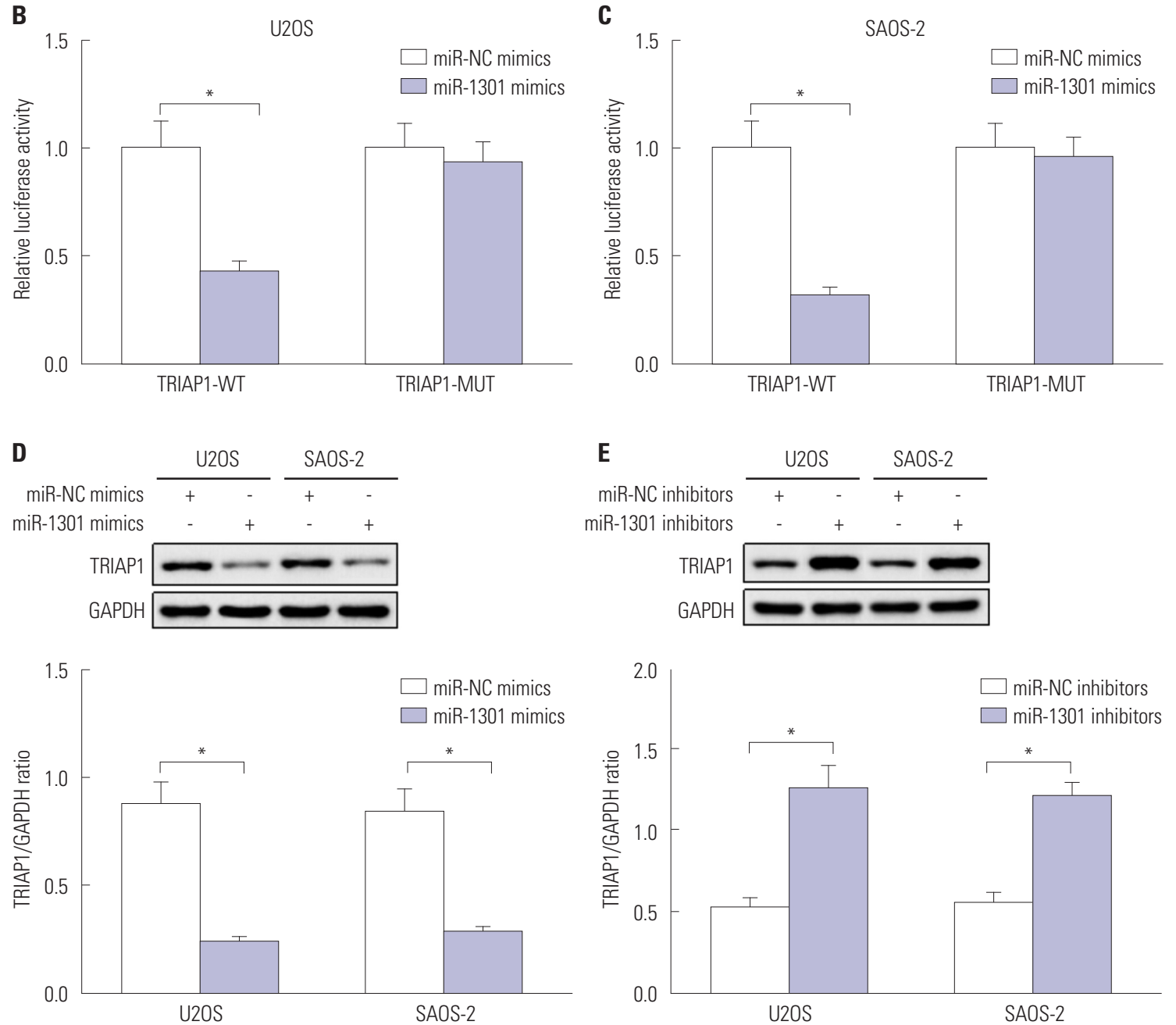

Fig. 5. TP53-regulated inhibitor of apoptosis 1 (TRIAP1) is a target of microRNA-1301 (miR-1301). (A) The binding sites between miR-1301 and TRIAP1 were predicted by the TargetScan online database, and luciferase reporter plasmids containing the wild-type (WT) or mutated (MUT) TRIAPT1 binding sites of miR-1301 were established. (B) The luciferase activity was measured in U20S cells co-transfected with TRIAP1-WT or TRIAP1-MUT luciferase reporter plasmids and miR-1301 mimics or miR-NC mimics. (C) The luciferase activity was examined in SAOS-2 cells co-transfected with TRIAP1-WT or TRIAP1MUT luciferase reporter plasmids and miR-1301 mimics or miR-NC mimics. (D) The expression of TRIAPT in U2OS and SAOS-2 cells transfected with miR1301 mimics or miR-NC mimics. (E) The expression of TRIAP1 in U2OS and SAOS-2 cells transfected with miR-1301 inhibitors or miR-NC inhibitors. (D and E) The + respensents Epirubicin or mimic was used to treat cells, while the meaning of - was opposite with $+.{ }^{*} p<0.05$. GAPDH, glyceraldehyde-3-phosphate dehydrogenase.

over, luciferase activity was dramatically reduced in the cells overexpressing miR-1301 in the TRIAP1 WT group, while it showed no significant change in the TRIAP1-MUT group (Fig. $5 \mathrm{~B}$ and $\mathrm{C}$ ), suggesting that TRIAP1 is a target of miR-1301. Furthermore, we also determined the effect of miR-1301 on expression of TRIAP1. The results showed a distinct suppression of TRIAP1 in miR-1301-upregulated cells, whereas inhi- bition of miR-1301 boosted it in U2OS and SAOS-2 cells (Fig. $5 \mathrm{D}$ and E), indicating that miR-1301 negatively regulates the expression of TRIAP1.

Epirubicin regulates TRIAP1 expression by modulating miR-1301

Finally, we also studied whether miR-1301/TRIAP1 axis is as- 
A

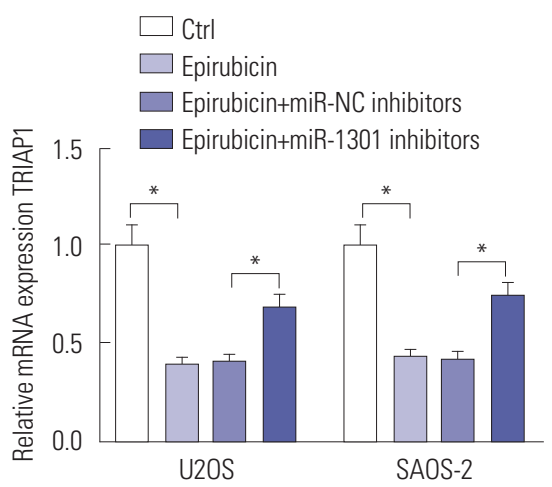

D

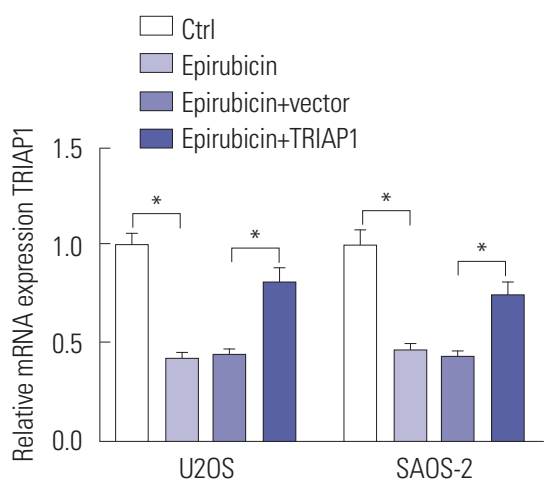

B
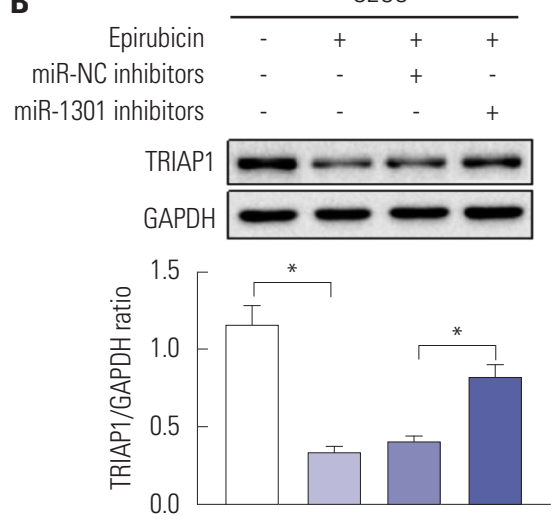

E
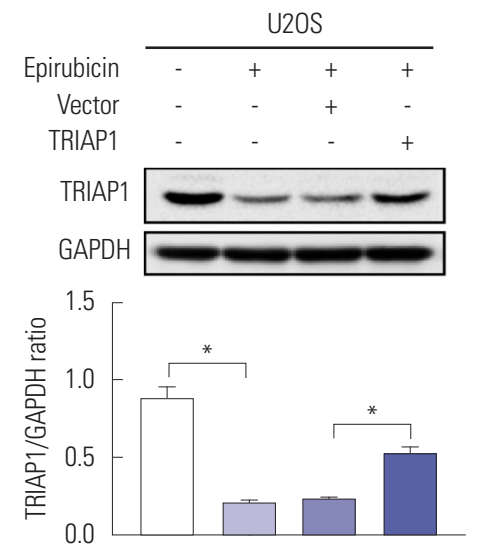

C
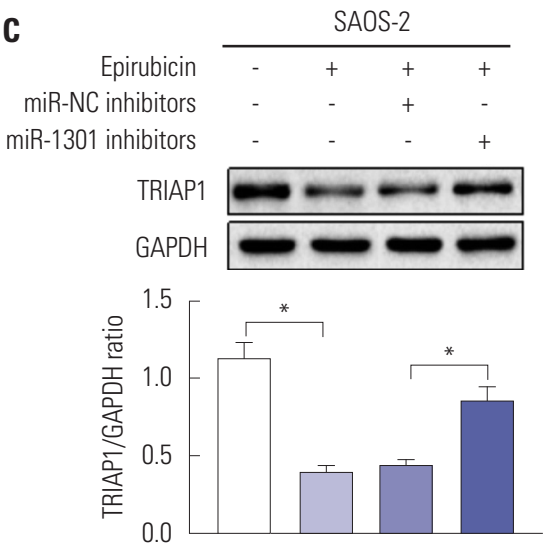

F

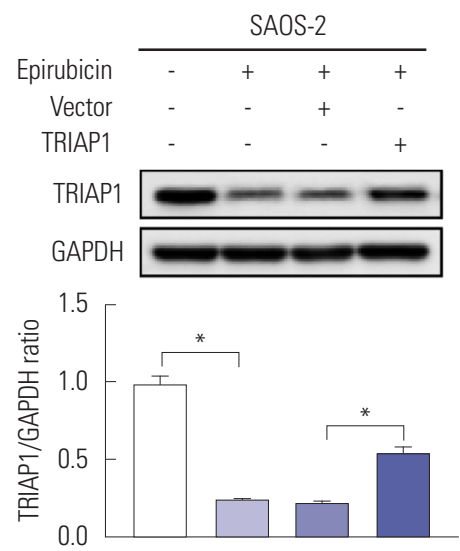

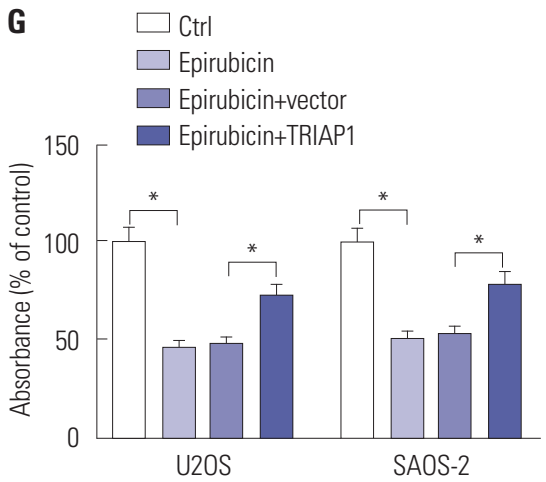

Fig. 6. Epirubicin regulates TP53-regulated inhibitor of apoptosis 1 (TRIAP1) expression by modulating microRNA-1301 (miR-1301). (A-C) U20S and SAOS2 cells were transfected with miR-NC inhibitors or miR-1301 inhibitors, and after $24-\mathrm{h}$ transfection, cells were treated with epirubicin $(1 \mu \mathrm{g} / \mathrm{mL})$ for $48 \mathrm{~h}$. Cells were divided into four groups: Ctrl, Epirubicin, Epirubicin+miR-NC inhibitors, or Epirubicin+miR-1301 inhibitors. (D-G) U2OS and SAOS-2 cells were transfected with vector or TRIAP1 overexpression plasmid (TRIAP1), and after 24-h transfection, cells were treated with epirubicin (1 $\mu \mathrm{g} / \mathrm{mL}$ ) for $48 \mathrm{~h}$. Cells were divided into four groups: Ctrl, Epirubicin, Epirubicin+vector, or Epirubicin+TRIAP1. The mRNA levels of TRIAP1 in U2OS and SAOS-2 cells were evaluated ( $A$ and $D$ ). Western blot was applied to examine the levels of TRIAP1 in U2OS (B and E) and SAOS-2 cells ( $C$ and F). (G) Cell viability was determined by Cell Counting Kit-8 assay. (B, C, E, and F) The + respensents Epirubicin or mimic was used to treat cells, while the meaning of - was opposite with + ${ }^{*} p<0.05$. GAPDH, glyceraldehyde-3-phosphate dehydrogenase.

sociated with epirubicin-mediated anti-osteosarcoma effects. qRT-PCR was subsequently employed to determine the mRNA levels of TRIAP1. We found that the mRNA levels of TRIAP1 were remarkably lower in epirubicin-treated osteosarcoma cells, which was attenuated by transfection of miR1301 inhibitors (Fig. 6A). Similarly, Western blot also demonstrated that epirubicin inhibited protein levels of TRIAP1 in
U2OS and SAOS-2 cells, which was rescued by miR-1301 knockdown (Fig. 6B and C). Subsequently, rescue-of-function was also performed to determine the effect of TRIAP1 upregulation on epirubicin-mediated TRIAP1 inhibition. Our data further indicated that overexpression of TRIAP1 abated lower expression of TRIAP1 induced by epirubicin at mRNA (Fig. 6D) and protein (Fig. 6E and F) levels. These data revealed 
that epirubicin repressed TRIAP1 expression by upregulating miR-1301. In addition, ectopic expression of TRIAP1 rescued the epirubicin-mediated decrease of cell viability (Fig. 6G). These results supported the hypothesis that miR-1301/ TRIAP1 may be implicated in epirubicin-mediated pro-apoptosis and anti-proliferation in osteosarcoma.

\section{DISCUSSION}

Epirubicin, an anti-tumor drug, has been used in the treatment of osteosarcoma. ${ }^{20}$ In the current study, we found that epirubicin curbed cell proliferation and induced apoptosis in osteosarcoma cells, which was in line with a previous study. ${ }^{20}$ Emerging evidence suggests that miR-1301 could serve as a tumor-suppressor in a variety of cancers. For example, Zhi, et al. ${ }^{23}$ disclosed that miR-1301 is downregulated in glioma tissues and cell lines and inhibits cell proliferation by targeting neuroblastoma ras viral oncogene homolog. miR-1301-3p is decreased in breast cancer tissues and cells, induces cell cycle G0/G1 phase arrest and apoptosis, and inhibits cell proliferation by targeting immature colon carcinoma transcript- $1 .{ }^{24}$ Here, we demonstrated that miR-1301 is expressed at low levels in osteosarcoma cells, suggesting that miR-1301 might contribute to the anti-osteosarcoma effect. Interestingly, the expression of miR-1301 showed an evident increase in cells after epirubicin exposure. These data indicated that miR-1301 may play an important role in osteosarcoma therapy in combination with epirubicin. Therefore, we subsequently investigated its role and the underlying molecular mechanisms in respect to epirubicin-mediated anti-osteosarcoma. Our study indicated that overexpression of miR-1301 hinders cell proliferation and promotes apoptosis in osteosarcoma cells, which is similar to the functions of epirubicin. To gain a deeper insight into the molecular mechanisms of epirubicin and miR1301 affecting apoptosis, the main regulatory molecules that govern the main basic mechanisms, such as the expression of apoptosis-related protein Bcl-2, Bax, cleaved-caspase-3, and poly (ADP-ribose) polymerase-1 (PARP1), were further examined. Bcl-2 is an anti-apoptosis protein, and dysregulation of Bcl-2-mediated apoptosis underlies a plethora of diseases. ${ }^{25}$ Bax is a pro-apoptotic member of the BCL-2 family. ${ }^{26}$ Also, detection of cleaved-caspase- 3 in cells is an important method for apoptosis induced by a wide variety of apoptotic signals. ${ }^{27,28}$ PARP1 is also a pro-apoptosis gene and could restore miR520-induced cell apoptosis. ${ }^{29}$ Our findings demonstrated that epirubicin and upregulation of miR-1301 both reduces the expression of Bcl-2 and increases the expressions of Bax, cleavedcaspase-3, and PARP1, further reflecting the miR-1301-mediated pro-apoptosis effect. Importantly, we also revealed that miR-1301 enhances the function of epirubicin-induced antiproliferation and pro-apoptosis in U2OS and SAOS-2 cells, indicating that miR-1301 increases the epirubicin effect. In con- trast, miR-1301 knockdown attenuated the effect of epirubicin on osteosarcoma cells. Therefore, we inferred that miR-1301 facilitates epirubicin-mediated chemosensitivity in osteosarcoma. miRNAs can regulate post-transcriptional gene expression and silence a broad set of targets genes. TRIAP1, a novel apoptosis inhibitor, has been shown to be upregulated in many types of cancers. ${ }^{30}$ Stable silencing of TRIAP1 induced late apoptosis in RPMI8226 cells. ${ }^{31}$ In addition, miR-18a inhibited ovarian cancer proliferation and induced apoptosis by targeting TRIAP $1 .^{32}$ miR-320b suppressed nasopharyngeal carcinoma cell proliferation and enhanced mitochondrial fragmentation and apoptosis by regulating TRIAP1. These data implied that TRIAP1 could be modulated by miRNAs. Here, we showed that miR-1301 repressed TRIAP1 expression by directly binding to the 3'UTR of TRIAP1. Moreover, the level of TRIAP1 was repressed by epirubicin in osteosarcoma cells, which was also rescued by introduction of miR-1301 inhibitors or TRIAP1 overexpression plasmid. Interestingly, restoration of TRIAP1 abolished the epirubicin-mediated cell proliferation inhibition. It is tempting to speculate that miR-1301 may participate in epirubicin-mediated chemosensitivity by targeting TRIAP1. Nevertheless, our study only investigated the role of miR1301/TRIAP1 axis in vitro, and it needs to be further confirmed in xenograft models. Moreover, the role of TRIAP1 alone in osteosarcoma should be addressed in the future.

Taken together, our data highlighted the pivotal role of miR1301 in epirubicin-mediated chemosensitivity in osteosarcoma. A novel miR-1301/TRIAP1 axis was first observed in osteosarcoma. Importantly, we found that an epirubicin-mediated miR-1301/TRIAP1 axis is involved in cell proliferation and apoptosis in osteosarcoma. Our findings provide powerful evidence of the molecular mechanism of chemosensitivity to epirubicin in osteosarcoma, which will help support clinical applications of a combination of epirubicin and miR-1301 in osteosarcoma.

\section{AUTHOR CONTRIBUTIONS}

Conceptualization: Lijun Yu and Min Meng. Data curation: Min Meng and Yun Bao. Formal analysis: Chao Zhang. Funding acquisition: Bei Gao and Rina Sa. Investigation: Wenyuan Luo and Lijun Yu. Methodology: Lijun Yu and Bei Gao. Project administration: Rina Sa and Lijun Yu. Resources: Wenyuan Luo. Software: Wenyuan Luo. Supervision: Bei Gao. Validation: Yun Bao. Visualization: Min Meng. Writing_original draft: Bei Gao and Lijun Yu. Writing_review \& editing: Lijun Yu and Yun Bao.

\section{ORCID iDs}

Lijun Yu https://orcid.org/0000-0001-8366-5179

Min Meng https://orcid.org/0000-0002-8133-3127

Yun Bao https://orcid.org/0000-0002-4019-6445

Chao Zhang https://orcid.org/0000-0003-2752-3373

Bei Gao https://orcid.org/0000-0001-5426-0252

Rina Sa https://orcid.org/0000-0003-1881-2077 
Wenyuan Luo https://orcid.org/0000-0003-0482-2231

\section{REFERENCES}

1. Moore DD, Luu HH. Osteosarcoma. Cancer Treat Res 2014;162: 65-92.

2. Biazzo A, De Paolis M. Multidisciplinary approach to osteosarcoma. Acta Orthop Belg 2016;82:690-8.

3. Harrison DJ, Geller DS, Gill JD, Lewis VO, Gorlick R. Current and future therapeutic approaches for osteosarcoma. Expert Rev Anticancer Ther 2018;18:39-50.

4. Fan XL, Cai GP, Zhu LL, Ding GM. Efficacy and safety of ifosfamide-based chemotherapy for osteosarcoma: a meta-analysis. Drug Des Devel Ther 2015;9:5925-32.

5. Luetke A, Meyers PA, Lewis I, Juergens H. Osteosarcoma treatment - where do we stand? A state of the art review. Cancer Treat Rev 2014;40:523-32.

6. Friebele JC, Peck J, Pan X, Abdel-Rasoul M, Mayerson JL. Osteosarcoma: a meta-analysis and review of the literature. Am J Orthop (Belle Mead NJ) 2015;44:547-53.

7. Petrioli R, Roviello G, Zanotti L, Roviello F, Polom K, Bottini A, et al. Epirubicin-based compared with docetaxel-based chemotherapy for advanced gastric carcinoma: a systematic review and meta-analysis. Crit Rev Oncol Hematol 2016;102:82-8.

8. Wu J, Xue X, Zhang B, Cao H, Kong F, Jiang W, et al. Enhanced antitumor activity and attenuated cardiotoxicity of Epirubicin combined with Paeonol against breast cancer. Tumour Biol 2016;37: 12301-13.

9. Liu ZL, Wang G, Shu Y, Zou PA, Zhou Y, Yin QS. Enhanced antitumor activity of epirubicin combined with cerulenin in osteosarcoma. Mol Med Rep 2012;5:326-30.

10. Basaran M, Bavbek ES, Saglam S, Eralp L, Sakar B, Atalar AC, et al. A phase II study of cisplatin, ifosfamide and epirubicin combination chemotherapy in adults with nonmetastatic and extremity osteosarcomas. Oncology 2007;72:255-60.

11. Acunzo M, Romano G, Wernicke D, Croce CM. MicroRNA and cancer--a brief overview. Adv Biol Regul 2015;57:1-9.

12. Waresijiang N, Sun J, Abuduaini R, Jiang T, Zhou W, Yuan H. The downregulation of miR-125a-5p functions as a tumor suppressor by directly targeting MMP-11 in osteosarcoma. Mol Med Rep 2016; 13:4859-64.

13. Bach DH, Hong JY, Park HJ, Lee SK. The role of exosomes and miRNAs in drug-resistance of cancer cells. Int J Cancer 2017;141: 220-30.

14. Wang J, Yang M, Li Y, Han B. The role of MicroRNAs in the chemoresistance of breast cancer. Drug Dev Res 2015;76:368-74.

15. Li Q, Liu M, Ma F, Luo Y, Cai R, Wang L, et al. Circulating miR-19a and miR-205 in serum may predict the sensitivity of luminal A subtype of breast cancer patients to neoadjuvant chemotherapy with epirubicin plus paclitaxel. PLoS One 2014;9:e104870.

16. Tan X, Fu Y, Chen L, Lee W, Lai Y, Rezaei K, et al. miR-671-5p inhibits epithelial-to-mesenchymal transition by downregulating
FOXM1 expression in breast cancer. Oncotarget 2016;7:293-307.

17. Zhang W, Qiao B, Fan J. Overexpression of miR-4443 promotes the resistance of non-small cell lung cancer cells to epirubicin by targeting INPP4A and regulating the activation of JAK2/STAT3 pathway. Pharmazie 2018;73:386-92.

18. Chen D, Liu D, Chen Z. Potential therapeutic implications of miRNAs in osteosarcoma chemotherapy. Tumour Biol 2017;39: 1010428317705762.

19. Wang F, Yu D, Liu Z, Wang R, Xu Y, Cui H, et al. MiR-125b functions as a tumor suppressor and enhances chemosensitivity to cisplatin in osteosarcoma. Technol Cancer Res Treat 2016;15: NP105-NP12.

20. Zhang Y, Hu H, Song L, Cai L, Wei R, Jin W. Epirubicin-mediated expression of miR-302b is involved in osteosarcoma apoptosis and cell cycle regulation. Toxicol Lett 2013;222:1-9.

21. Wang L, Hu K, Chao Y. MicroRNA-1301 inhibits migration and invasion of osteosarcoma cells by targeting BCL9. Gene 2018;679: 100-7.

22. Li Y, Tang X, He Q, Yang X, Ren X, Wen X, et al. Overexpression of mitochondria mediator gene TRIAP1 by miR-320b loss is associated with progression in nasopharyngeal carcinoma. PLoS Genet 2016;12:e1006183.

23. Zhi T, Jiang $\mathrm{K}$, Zhang C, Xu X, Wu W, Nie E, et al. MicroRNA-1301 inhibits proliferation of human glioma cells by directly targeting N-Ras. Am J Cancer Res 2017;7:982-98.

24. Peng X, Yan B, Shen Y. MiR-1301-3p inhibits human breast cancer cell proliferation by regulating cell cycle progression and apoptosis through directly targeting ICT1. Breast Cancer 2018;25:742-52.

25. Kvansakul M, Caria S, Hinds MG. The Bcl-2 family in host-virus interactions. Viruses 2017;9. pii: E290.

26. Raemy E, Martinou JC. Involvement of cardiolipin in tBID-induced activation of BAX during apoptosis. Chem Phys Lipids 2014;179:70-4.

27. Choudhary GS, Al-Harbi S, Almasan A. Caspase-3 activation is a critical determinant of genotoxic stress-induced apoptosis. Methods Mol Biol 2015;1219:1-9.

28. Elmore S. Apoptosis: a review of programmed cell death. Toxicol Pathol 2007;35:495-516.

29. Dong X, Yang L, Wang H. miR-520 promotes DNA-damage-induced trophoblast cell apoptosis by targeting PARP1 in recurrent spontaneous abortion (RSA). Gynecol Endocrinol 2017;33:274-8.

30. Adams C, Cazzanelli G, Rasul S, Hitchinson B, Hu Y, Coombes RC, et al. Apoptosis inhibitor TRIAP1 is a novel effector of drug resistance. Oncol Rep 2015;34:415-22.

31. Fook-Alves VL, de Oliveira MB, Zanatta DB, Strauss BE, Colleoni GW. TP53 Regulated Inhibitor of Apoptosis 1 (TRIAP1) stable silencing increases late apoptosis by upregulation of caspase 9 and APAF1 in RPMI8226 multiple myeloma cell line. Biochim Biophys Acta 2016;1862:1105-10.

32. Liu P, Qi X, Bian C, Yang F, Lin X, Zhou S, et al. MicroRNA-18a inhibits ovarian cancer growth via directly targeting TRIAP1 and IPMK. Oncol Lett 2017;13:4039-46. 